Acta Crystallographica Section F

Structural Biology

and Crystallization

Communications

ISSN 1744-3091

Leonard M. Thomas, Angelica R. Harper, Whitney A. Miner, Helen O. Ajufo, Katie M.

Branscum, Lydia Kao and

Paul A. Sims*

Department of Chemistry and Biochemistry University of Oklahoma, 101 Stephenson Parkway, Norman, OK 73019-5251, USA

Correspondence e-mail: psims@ou.edu

Received 17 May 2013

Accepted 1 June 2013

PDB Reference: AdhP-NAD complex, 4gkv

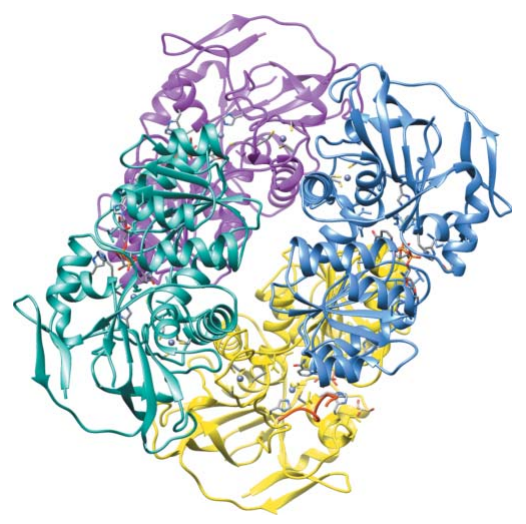

C 2013 International Union of Crystallography All rights reserved

\section{Structure of Escherichia coli AdhP (ethanol- inducible dehydrogenase) with bound NAD}

The crystal structure of AdhP, a recombinantly expressed alcohol dehydrogenase from Escherichia coli K-12 (substrain MG1655), was determined to $2.01 \AA$ resolution. The structure, which was solved using molecular replacement, also included the structural and catalytic zinc ions and the cofactor nicotinamide adenine dinucleotide (NAD). The crystals belonged to space group $P 2_{1}$, with unit-cell parameters $a=68.18, b=118.92, c=97.87 \AA$, $\beta=106.41^{\circ}$. The final $R$ factor and $R_{\text {free }}$ were 0.138 and 0.184 , respectively. The structure of the active site of AdhP suggested a number of residues that may participate in a proton relay, and the overall structure of AdhP, including the coordination to structural and active-site zinc ions, is similar to those of other tetrameric alcohol dehydrogenase enzymes.

\section{Introduction}

Alcohol dehydrogenase (ADH) has held a special place in the minds of mechanistic enzymologists ever since the classical work of Westheimer and coworkers revealed the stereospecific transfer of hydrogen from the alcohol to the nicotinamide ring of the cofactor NAD (Fisher et al., 1953). Relatively recently, a 'new' alcohol dehydrogenase was discovered in Escherichia coli when it was found that $\sim 17 \mathrm{~m} M$ ethanol induced the expression of an enzyme whose properties were different from the class III ADH that had previously been characterized in E. coli (Shafqat et al., 1999). Amino-acid sequencing of the protein revealed that it was the product of the adhP gene (Shafqat et al., 1999; Blattner et al., 1997).

Similar to other ADHs, the $a d h P$ gene product (AdhP) catalyzes NAD/NADH-linked oxidation-reduction reactions of short-chain alcohols and aldehydes (or ketones). The enzyme allows E. coli to excrete ethanol when conditions require mixed-acid fermentation or to utilize ethanol as an energy source (Nosova et al., 1997). Previous structures of AdhP have been reported (Karlsson et al., 2003); however, the coordinate files for these structures were unavailable, which limited opportunities for comparison with the present structure. Nonetheless, the structure reported here and the previous structure of the NAD-bound enzyme (Karlsson et al., 2003) show, not surprisingly, a number of similarities but also some distinct differences, as discussed below.

\section{Materials and methods}

\subsection{Macromolecule production}

The adhP gene from E. coli was cloned into the pET101/D-TOPO vector from Invitrogen. The resulting plasmid (pET-AdhP) was transformed into expression cells [E. coli BL21 (DE3)]. A $50 \mathrm{ml}$ starter culture was grown for $\sim 9 \mathrm{~h}$ and was then split into two 21 flasks that each contained $1 \mathrm{lLB}$ medium plus $0.4 \mathrm{~g}^{-1}$ ampicillin and $16 \mathrm{~g} \mathrm{l}^{-1}$ lactose. Growth at $310 \mathrm{~K}$ and $200 \mathrm{rev} \mathrm{min}^{-1}$ continued for an additional $20 \mathrm{~h}$. Harvested cells were stored for around one week at $253 \mathrm{~K}$ and were then added to lysis buffer ( $0.05 \mathrm{M}$ sodium phosphate, $0.3 M \mathrm{NaCl}, 0.01 M$ imidazole $\mathrm{pH} 8$ ) containing $0.05 \mathrm{~g}$ lysozyme, $0.04 \mathrm{~g}$ PMSF and $\sim 1 \mathrm{mg}$ DNaseI. The mixture was shaken at $100 \mathrm{rev} \mathrm{min}^{-1}$ for $2 \mathrm{~h}$ and then centrifuged at $15000 \mathrm{~g}$ for $15 \mathrm{~min}$. The clarified supernatant was added to an Ni-NTA column and AdhP was 
Table 1

Macromolecule-production information.

\begin{tabular}{|c|c|}
\hline Source organism & E. coli $\mathrm{K}-12$ (substr. MG1655) \\
\hline DNA source & $\begin{array}{l}\text { Genomic DNA isolated from E. coli } \mathrm{K}-12 \text { (substr. } \\
\text { MG1655) }\end{array}$ \\
\hline Forward primer & CACCATGAAGGCTGCAGTTGTTAC \\
\hline Reverse primer & $\begin{array}{l}\text { GTGACGGAAATCAATCACCATGC (no stop } \\
\text { codon; read in-frame with the vector to add a V5 } \\
\text { epitope and a } \mathrm{His}_{6} \mathrm{tag} \text { ) }\end{array}$ \\
\hline Cloning vector & pET101/D-TOPO \\
\hline Expression vector & $\begin{array}{l}\text { pET101/D-TOPO (subsequently designated } \\
\text { pET-AdhP) }\end{array}$ \\
\hline Expression host & E. coli BL21 Star (DE3) \\
\hline $\begin{array}{l}\text { Complete amino-acid sequence } \\
\text { of the construct produced }\end{array}$ & $\begin{array}{l}\text { MKAAVVTKDHHVDVTYKTLRSLKHGEALLK- } \\
\text { MECCGVCHTDLHVKNGDFGDKTGVILGH- } \\
\text { EGIGVVAEVGPGVTSLKPGDRASVAWFYE- } \\
\text { GCGH CEYCNSGNETLCRSVKNAGYSVDG- } \\
\text { GMAEECIVVADYAVKVPDGLDSAAASSIT- } \\
\text { CAGVTTYKAVKLSKIRPGQWIAIYGLGGL- } \\
\text { GNLALQYAKN VFNAKVIAIDVNDEQLKL- } \\
\text { ATEMGADLAINSHTEDAAKIVQEKTGGAH- } \\
\text { AAVVTAVAKAAFNSAVDAVRAGGRVVAVG- } \\
\text { LPPESMSLDIPRLVLD GIEVVGSLVGTRQD- } \\
\text { LTEAFQFAAEGKVVPKVALRPLADINTIFT- } \\
\text { EMEEGKIRGRMVIDFRHKGELNSKLEGKP- } \\
\text { IPNPLLGLDSTRTGHHHHHH }\end{array}$ \\
\hline
\end{tabular}

eluted with a $50 \mathrm{ml} 0.02-0.25 \mathrm{M}$ imidazole gradient. Macromoleculeproduction information is presented in Table 1 .

\subsection{Crystallization}

Initial broad crystallization screens were set up using a Mosquito nanolitre liquid handler (TTP Labtech Inc.). Promising conditions were optimized manually using the hanging-drop vapor-diffusion method in 24-well plates. The protein concentration was $64 \mathrm{~g} \mathrm{l}^{-1}$ in a

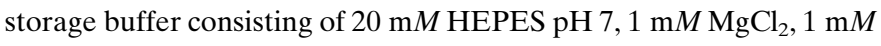
$\mathrm{NaN}_{3}$. The final reservoir conditions were $0.2 \mathrm{M} \mathrm{Na}_{2} \mathrm{SO}_{4}, 0.1 M$ bistris propane $\mathrm{pH} 8.5,10 \mathrm{~m} M$ NAD, 20\% PEG 3350. Single crystals were harvested using a nylon cryoloop and successively transferred into cryoprotectant solutions with increasing concentrations of glycerol $(5,10,15$ and $20 \%)$ but that otherwise matched the reservoir conditions. After the last transfer, the crystals were flash-cooled to $100 \mathrm{~K}$ using an Oxford Cryosystems 700 Series Cryostream.

\subsection{Data collection and processing}

X-ray diffraction data were collected on an R-AXIS IV $^{++}$imageplate detector (Rigaku Americas Inc.) using $\mathrm{Cu} K \alpha$ radiation from

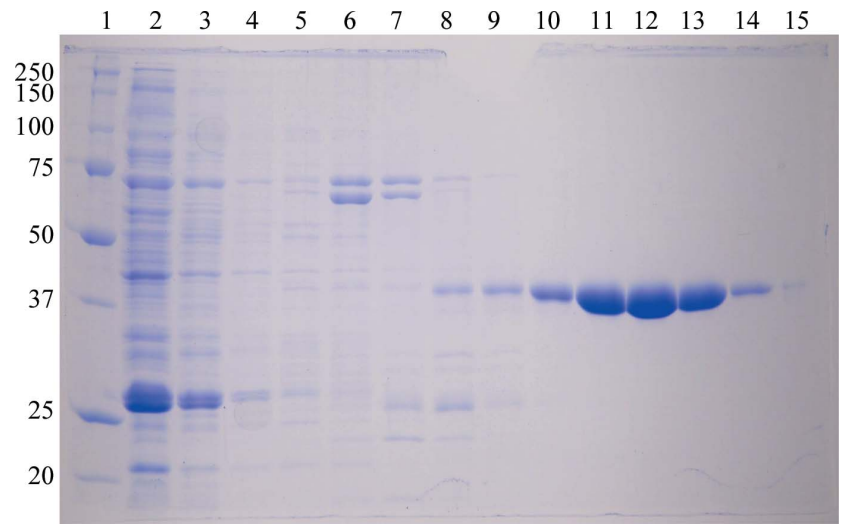

Figure 1

Coomassie-stained gel from the SDS-PAGE analysis of fractions eluted from the Ni-NTA column during the purification of AdhP. Lanes are as follows: 1, molecular-mass ladder (labeled in $\mathrm{kDa}$ ); 2 , load flowthrough; 3, wash flowthrough; $4-15$, fractions $1-12$, respectively. Fractions $7-10$ were pooled, buffer exchanged/ concentrated and used in crystallization trials.
Table 2

Data-collection and processing statistics.

Values in parentheses are for the outermost shell.

\begin{tabular}{ll}
\hline Wavelength $(\AA)$ & 1.5418 \\
Temperature $(\mathrm{K})$ & 100 \\
Space group & $P 2_{1}$ \\
Unit-cell parameters $\left(\AA{ }^{\circ}{ }^{\circ}\right)$ & $a=68.18, b=118.92, c=97.87$, \\
& $\alpha=\gamma=90.0, \beta=106.41$ \\
Mosaicity $\left({ }^{\circ}\right)$ & 1.12 \\
Resolution range $(\AA)$ & $32.6-2.01(2.12-2.01)$ \\
Total No. of reflections & 321023 \\
No. of unique reflections & 99085 \\
Completeness $(\%)$ & $99.5(98.7)$ \\
Multiplicity & $3.2(3.0)$ \\
$\langle I / \sigma(I)\rangle$ & $11.7(4.7)$ \\
$R_{\text {r.i.m. }}$ & $0.079(0.247)$ \\
Overall $B$ factor from Wilson plot $\left(\AA^{2}\right)$ & 13.2 \\
\hline
\end{tabular}

a Rigaku RU-H3R rotating-anode generator. Image data were processed using MOSFLM (Leslie \& Powell, 2007), with scaling and averaging performed using SCALA (Evans, 2006) in CCP4 (Winn et al., 2011). Data-processing statistics are presented in Table 2.

\subsection{Structure solution and refinement}

The crystal structure of AdhP was solved via molecular replacement using Phaser (McCoy et al., 2007). The structure of Brucella melitensis alcohol dehydrogenase (PDB entry 3meq; Seattle Structural Genomics Center for Infectious Disease, unpublished work) was used as the initial model. The structure was refined and water molecules were added using PHENIX (Adams et al., 2010; phenix.refine v.1.8_1069). Model building was performed using Coot (Emsley et al., 2010). MolProbity (Chen et al., 2010) was used for validiation analysis and UCSF Chimera (Pettersen et al., 2004) was used to construct the molecular-graphics images in Figs. 2 and 3. Refinement statistics are summarized in Table 3.

\section{Results and discussion}

In the initial report that described the purification and characterization of AdhP (Shafqat et al., 1999), the authors stated that wildtype AdhP bound to an Ni-NTA column; this binding was attributed to the presence of five histidine residues (only two of which were consecutive) that were located near the N-terminus of the protein. In addition to the wild-type sequence, the construct reported here contains 32 additional amino-acid residues appended to the $\mathrm{C}$-terminus. These extra residues include a V5 epitope and a $\mathrm{His}_{6}$ tag. As a consequence, the protein bound tightly to the Ni-NTA column and eluted relatively late in the gradient $(\sim 0.23 \mathrm{M}$ imidazole $)$ in a highly pure form (Fig. 1).

A ribbon representation showing the tetrameric structure of AdhP is presented in Fig. 2. The structure of AdhP included a short peptide, which is shown in orange in the lower right-hand portion of Fig. 2. This peptide is part of the 32 extra amino-acid residues (Pro348Leu357) that were incorporated into the construct. In all four subunits the electron density was disordered after the last naturally occurring amino acid (His336) and thus the subunit that contributed the extra density that corresponds to the above fragment is unknown.

The group that reported the previous structures of AdhP provided a number of comparisons with other ADH structures (Karlsson et al., 2003). One of the previous structures of AdhP was of the apoenzyme and the other was of the holoenzyme (with bound NAD); in both cases the space group of the crystals was $P 2_{1} 2_{1} 2_{1}$ (Karlsson et al., 2003). It is expected that the previous structures and the present structure are highly similar, but the presence of the extra 32 amino- 
acid residues at the $\mathrm{C}$-terminus of the present structure and the observation that the crystals that led to the present structure belong to space group $P 2_{1}$ may in part explain some of the noted differences. For example, in the previous structure of the holoenzyme three of the four subunits had bound NAD, and in these subunits the coordination to the catalytic zinc ion was of the 'classical' type in which two cysteines, a histidine and a water coordinate the zinc ion; the subunit that did not have NAD bound exhibited a different coordination of the active-site zinc ion such that the coordinating residues were the same but the water was replaced by a glutamate residue (Karlsson et $a l ., 2003)$. In the present structure all four subunits have bound NAD,

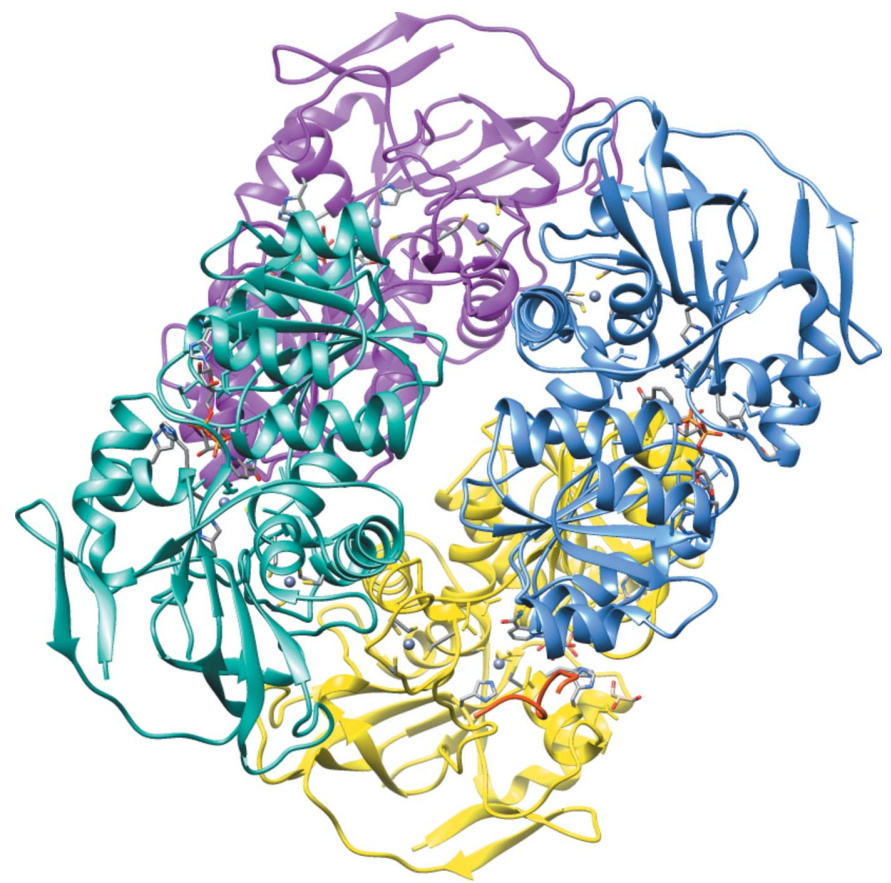

Figure 2

Ribbon representation of the AdhP tetramer looking down the twofold-rotation axis. Zinc ions are shown as gray spheres and NAD, glycerol, $\mathrm{Zn}^{2+}$-coordinating residues and selected active-site residues are shown in stick form. The short peptide shown in orange at the lower right of the figure corresponds to part of the C-terminus, as discussed in the text.

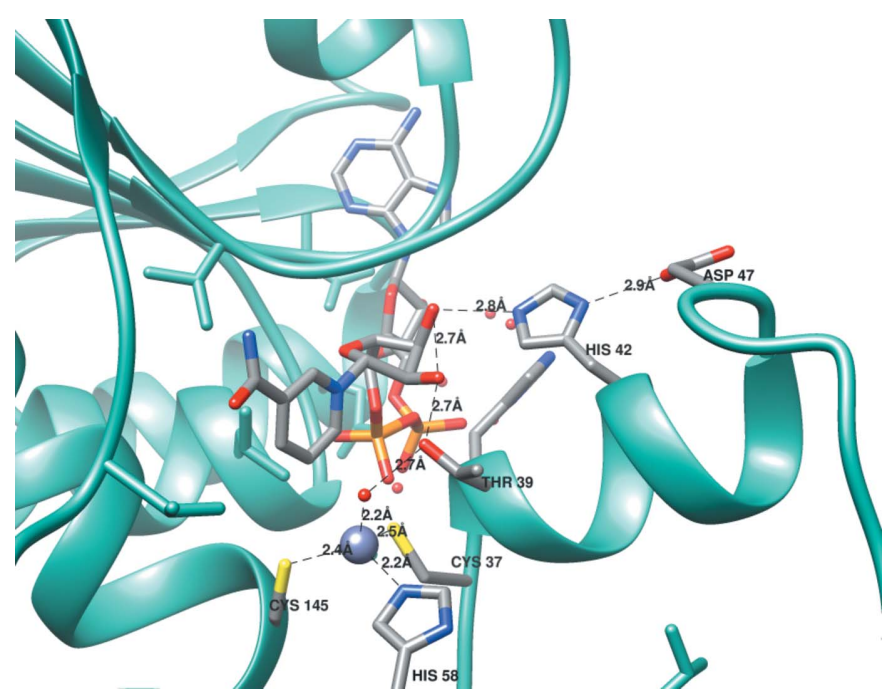

Figure 3

Close-up view of the active site of AdhP showing the coordinating residues of the catalytic zinc ion and the residues/moieties that may be part of a proton relay.
Table 3

Structure-refinement statistics.

\begin{tabular}{ll}
\hline Resolution range $(\AA)$ & $32.690-2.008$ \\
Completeness (\%) & 99.5 \\
$\sigma$ Cutoff & 2 \\
No. of reflections, working set & 94131 \\
No. of reflections, test set & 4954 \\
Final $R_{\text {cryst }}$ & 0.138 \\
Final $R_{\text {free }}$ & 0.184 \\
No. of non-H atoms & \\
$\quad$ Protein & 10018 \\
$\quad$ Ligand & 196 \\
$\quad$ Solvent & 1730 \\
$\quad$ Total & 11944 \\
R.m.s. deviations & \\
$\quad$ Bonds $(\AA)$ & 0.007 \\
$\quad$ Angles $\left({ }^{\circ}\right)$ & 1.132 \\
Average $B$ factors ( $\left.\AA^{2}\right)$ & 10.3 \\
$\quad$ Protein & 9.2 \\
$\quad$ Ligand & 21.0 \\
$\quad$ Water & \\
Ramachandran plot & 97.0 \\
$\quad$ Favoured regions $(\%)$ & 3.0 \\
$\quad$ Additionally allowed regions $(\%)$ & \\
\hline
\end{tabular}

and in each of the subunits the coordination scheme of the active-site zinc ion was of the classical type (Fig. 3).

The structure of the AdhP active site also suggests the identities of moieties/residues that participate in a proton relay: Asp47, His42, the $2^{\prime}-\mathrm{OH}$ and $3^{\prime}-\mathrm{OH}$ of the ribose attached to the nicotinamide ring of NAD, and Thr39. A similar relay has been proposed in horse liver ADH (Luo \& Bruice, 2001) and site-directed mutagenesis experiments are planned to test this hypothesis in the case of AdhP.

This work was supported in part by a Center of Biomedical Research Excellence (CoBRE) award from the National Institute of General Medical Sciences (NIGMS) of the National Institutes of Health (P20GM103640) and by funding from the University of Oklahoma. The molecular-graphics program UCSF Chimera was developed by the Resource for Biocomputing, Visualization and Informatics at the University of California, San Francisco with support by NIGMS P41-GM103311.

\section{References}

Adams, P. D. et al. (2010). Acta Cryst. D66, 213-221.

Blattner, F. R. et al. (1997). Science, 277, 1453-1462.

Chen, V. B., Arendall, W. B., Headd, J. J., Keedy, D. A., Immormino, R. M., Kapral, G. J., Murray, L. W., Richardson, J. S. \& Richardson, D. C. (2010). Acta Cryst. D66, 12-21.

Emsley, P., Lohkamp, B., Scott, W. G. \& Cowtan, K. (2010). Acta Cryst. D66, 486-501.

Evans, P. (2006). Acta Cryst. D62, 72-82.

Fisher, H. F., Conn, E. E., Vennesland, B. \& Westheimer, F. H. (1953). J. Biol. Chem. 202, 687-697.

Karlsson, A., El-Ahmad, M., Johansson, K., Shafqat, J., Jörnvall, H., Eklund, H. \& Ramaswamy, S. (2003). Chem. Biol. Interact. 143-144, 239-245.

Leslie, A. G. W. \& Powell, H. R. (2007). Evolving Methods for Macromolecular Crystallography, edited by R. J. Read \& J. L. Sussman, pp. 41-51. Dordrecht: Springer.

Luo, J. \& Bruice, T. C. (2001). J. Am. Chem. Soc. 123, 11952-11959.

McCoy, A. J., Grosse-Kunstleve, R. W., Adams, P. D., Winn, M. D., Storoni, L. C. \& Read, R. J. (2007). J. Appl. Cryst. 40, 658-674.

Nosova, T., Jousimies-Somer, H., Kaihovaara, P., Jokelainen, K., Heine, R. \& Salaspuro, M. (1997). Alcohol. Clin. Exp. Res. 21, 489-494.

Pettersen, E. F., Goddard, T. D., Huang, C. C., Couch, G. S., Greenblatt, D. M., Meng, E. C. \& Ferrin, T. E. (2004). J. Comput. Chem. 25, 1605-1612.

Shafqat, J., Höög, J. O., Hjelmqvist, L., Oppermann, U. C., Ibáñez, C. \& Jörnvall, H. (1999). Eur. J. Biochem. 263, 305-311.

Winn, M. D. et al. (2011). Acta Cryst. D67, 235-242. 